\title{
Idealisierung, Modellierung, Übersteigerung. Zum deutschen Brautbrief des 18. Jahrhunderts
}

Idéalisation, modélisation, exagération. Réflexions sur la «lettre

fiancée » du XVIII siècle

Idealisation, Modeling, Exaggeration. Reflexions on the German "letter to a bride" in the $18^{\text {th }}$ century

Karl Heinz Götze

URL : http://journals.openedition.org/ceg/957

DOI : $10.4000 /$ ceg. 957

ISSN : 2605-8359

Éditeur

Presses Universitaires de Provence

Édition imprimée

Date de publication : 30 mai 2016

Pagination : 183-192

ISBN : 979-10-320-0067-0

ISSN : 0751-4239

\section{Référence électronique}

Karl Heinz Götze, «Idealisierung, Modellierung, Übersteigerung. Zum deutschen Brautbrief des 18. Jahrhunderts », Cahiers d'Études Germaniques [Online], 70 | 2016, Online erschienen am: 17 Dezember 2017, abgerufen am 26 November 2020. URL: http://journals.openedition.org/ceg/957 DOI : https://doi.org/10.4000/ceg.957 


\title{
Idealisierung, Modellierung, Übersteigerung Zum deutschen Brautbrief des 18. Jahrhunderts
}

\author{
Karl Heinz GÖTZE \\ Aix-Marseille Université
}

Ausgangspunkt meines Interesses für Brautbriefe war ein Befremden, das ich bei der Lektüre von Autoren des 18. und 19. Jahrhunderts häufig empfunden habe, das Befremden darüber, dass Autoren, deren literarische Werke ich überaus schätze, so seltsame, schulmeisterlich anmutende Briefe an ihre Bräute geschrieben haben, Briefe, die heute nicht mehr geschrieben werden könnten ohne der Lächerlichkeit anheim zu fallen, während Werke der gleichen Autoren durchaus über die Zeiten hinweg Bestand haben.

Es geht mir dabei nicht um „Entlarvung“ oder Anklage, darum, diese oder jene deutsche Geistesgröße oder gar alle deutschen Geistesgrößen der Zeit des heimlichen Machismus zu überführen. Das ist reichlich - und häufıg reichlich unhistorisch - geschehen. Ich möchte einfach nur Erklärungen dafür finden, warum viele dieser Brautbriefe uns so seltsam klingen. $\mathrm{Zu}$ dem Zwecke ist es vielleicht nützlich, in einem ersten, vorwissenschaftlichen Anlauf das Gefühl darauf zu befragen, was denn da seltsam anmutet. Ich rede natürlich von meinem Gefühl, aber ich glaube, man kann dessen Eindruck cum grano salis auf heutige Leser beiderlei Geschlechts verallgemeinern.

Zumindest drei Elemente lassen sich auf Anhieb dingfest machen. Zunächst fast immer eine übersteigerte, heute befremdliche Gefühligkeit. Im Jargon der gegenwärtigen Briefkultur gesprochen: Die Briefe sind voller „Smilys“, die in der Häufung fast immer stereotyp wirken. Dass die Smilys häufig Tränen in den Augen haben, ändert wenig. Dazu passt die Idealisierung des Briefpartners, der auf der Seite der Braut Grenzen der Schicklichkeit gesetzt sind, auf Seiten des Bräutigams aber, so will es scheinen, gar keine. Die Bräute sind in diesen Briefen zur Anbahnung eines „Geschlechtsgemeinschaft“, mit Kant verstanden als der „wechselseitige Gebrauch, den ein Mensch von eines anderen Geschlechtsorganen und Vermögen macht" ${ }^{1}$ allemal Engel und die haben bekanntlich keine Geschlechtsorgane. Zum Gefühlsüberschwang und zur Verhimmelung der Braut will aber schlecht die Schulmeisterei passen, dazu, dass kaum ein gebildeter Bräutigam darauf verzichten mag, eine Art epistoläres Fernstudium zu organisieren. Idealisierung, Modellierung und Übersteigerung

1. Immanuel Kant, Die Metaphysik der Sitten, § 24. 
sind, so meine These, zentrale Charakteristika der Brautbriefe des 18. und 19. Jahrhunderts. Wie kommt das?

Um dieser Frage nachgehen zu können, bedarf es freilich notwendig einiger sehr knapper Überlegungen zur Gattung der Brautbriefe, einer näheren Bestimmung der Zeit, in der sie geschrieben wurden und dann natürlich eines konkreten Blicks auf entsprechende Briefwechsel.

Zur Frage der Gattung: Brautbriefe sind nicht einfach Liebesbriefe, die übrigens im Gegensatz zu dem, was man spontan meinen möchte, auch nicht immer schon geschrieben wurden von allen, die schreiben und begehren konnten. Brautbriefe sind in einer älteren Bedeutung Briefe, in denen sich die Männer (Brautvater, Bräutigam, evtl. Vater des Bräutigams) über die Heiratsbedingungen, vor allem über die Mitgift, verständigten. Diese Briefe wiesen bis ins 18. Jahrhundert hinein auch in der Form Züge des juristischen Briefes auf, denn schließlich ging es ja auch um einen Akt von quasi-rechtlicher Bedeutung. Diese Praxen sind heute in Frankreich wie in Deutschland weitgehend verschwunden, wenn man von Einwandererkulturen einmal für einen Moment absieht: „Verlobungen sind überflüssig geworden, seitdem Liebespaare zusammenleben dürfen und nicht einmal mehr für die Ehe die elterliche Zustimmung nötig ist. Früher regelte die Verlobung die Übergabe der Tochter aus der Hand des einen Mannes, des Vaters, in die des anderen, des künftigen Schwiegersohnes. Damit war die Kontinuität der familialen Bindung der Frau gesichert. Von dem Augenblick an, da der Bräutigam das doppelte Jawort, der Braut und des Brautvaters, erhalten hatte, trat er als Erzieher seiner künftigen Frau an die Stelle des Vaters.“ ${ }^{2}$ Das weitgehende Verschwinden der Verlobung vollzog sich allmählich und keineswegs in allen sozialen Klassen im gleichen Takt. Das gilt auch für das notwendige „Ja“ des Vaters, das erst allmählich neben dem „Ja“ der Braut alle rechtliche Bedeutung verliert. Lange Zeit galt es, einerseits mit den Brautbriefen die Braut emotional zu gewinnen, andererseits aber auch, die Ordnung des künftigen Hausstands vorzubereiten, unter den Bedingungen der Distanz eine nicht eben einfach ins Werk zu setzende Aufgabe.

Brautbriefe sind also nicht einfach Liebesbriefe, sondern Liebesbriefe in der Perspektive einer künftigen Heirat, gleich, ob diese schließlich zustande kommt, gleich auch, ob man, wie etwa im Falle Mörikes an der Ernsthaftigkeit der Heiratsabsicht eines Partners, meist des Mannes, zweifeln kann. Es zählt die angenommene Absicht, stelle sie sich auch post festum als fiktiv heraus.

Der Name der „Brautbriefe“ kann auch nicht Briefen an Dritte über die Braut zukommen. Einer der schönsten der ganzen deutschen Literaturgeschichte ist der, den Lichtenberg im Herbst 1782 kurz nach dem Tod der „Jungfer Stechhard“ über sein Verhältnis zur „kleinen Stechardin“ schrieb, die er schon im zarten Alter

2. Deutsche Briefe 1750-1950, hrsg. von Gert Mattenklott/Hannelore Schlaffer/Heinz Schlaffer, Frankfurt a.M., S. Fischer, 1988, S. 297. 
von 11 Jahren zu sich ins Haus genommen hatte und die bald auch seine geliebte Bettgefährtin wurde, bevor sie fünf Jahre später verstarb. Walter Benjamin hat diesen Brief als Zeugnis „der wahren Humanität“ in seine Epistelsammlung Deutsche Menschen aufgenommen ${ }^{3}$, Gert Hofmann hat der kleinen Stechardin einen wunderbaren, leider zu wenig bekannten Roman gewidmet. ${ }^{4}$ Der Fall ist in vieler Hinsicht interessant. Nebenbei auch deshalb, weil man hier sieht, dass in Brautfragen zwar die väterliche Autorität an Bedeutung verliert, dafür aber die staatliche an Bedeutung gewinnt. Man stelle sich vor, in unserer Zeit würde ein berühmter Göttinger Physikprofessor dabei ertappt, dass er mit einem dreizehnjährigen Mädchen das Bett teilt... Interessant ist der Fall aber für uns auch deshalb, weil er an eine elementare Voraussetzung für Verlobungskorrespondenz erinnert, die im 18. Jahrhundert noch keineswegs durchgehend gegeben ist: die Partner, von denen zumindest der weibliche Teil häufig keinerlei Schulbildung erfahren hatte, müssen die Schrift beherrschen. Das war bei der Stechardin nur rudimentär der Fall.

Ein anderer Aspekt ist nicht so leicht greifbar und noch schwerer beweisbar, aber er dürfte dennoch eine Rolle spielen: Lichtenbergs Brief rührt auch heute noch, weil ihm alle Überspannung fehlt, alle Tränenseligkeit, alle Rührungsabsicht, so wie übrigens auch dem berühmten Brief, den Lessing nach dem Tod seiner Frau an Eschenburg schrieb. ${ }^{5}$ Klar, Lichtenberg und Lessing standen der Empfindsamkeit höchst kritisch gegenüber und haben kein Hehl aus ihren Gründen gemacht. Mir scheint aber, es gibt auch sozusagen gattungsimmanente Gründe dafür, dass die Brautbriefe ein bevorzugter Ort gehobener, ja hochgespannter Empfindsamkeit sind: Sie werden geschrieben aus einer Situation künstlichen Gefühlsstaus, bewirkt durch das Distanzgebot. ${ }^{6}$ Ja, es ist hier auch von Sexualität die Rede, gestauter Sexualität, die die Brautleute in vielfacher Hinsicht lockte und zugleich bedrohte. Zumindest im Falle der Braut war der Verzicht auf voreheliche Sexualität unabdingbar für das soziale Überleben. Vergessen wir auch nicht die religiösen Wurzeln der deutschen Empfindsamkeit, sublimiert in einem Tugenddiskurs, der sich der adeligen Lebenspraxis entgegen stellte und insofern eine zentrale identitäre Funktion hatte. Libertinage war unter diesen Gesichtspunkten für die bürgerlich gestimmte deutsche Intelligenz nicht nur Sünde, sondern vor allem auch so etwas wie Selbstaufgabe.

Es scheint mir kaum ein Zufall zu sein, dass nicht nur Briefe über die Braut, sondern auch Briefe zwischen Partnern in "Geistesehe“" (so einst die paradoxe Bezeichnung für ein nicht verheiratet zusammenlebendes Paar) ohne die Überspanntheit der Briefe zwischen Brautleuten sind. Goethes Briefwechsel

3. Walter Benjamin, Deutsche Menschen. Eine Folge von Briefen, in ders., Gesammelte Schriften, Bd. IV, 1, hrsg. von Tillman Rexroth, Frankfurt a.M., Suhrkamp,1980, S. 149-233, hier S. 153 f.

4. Gert Hofmann, Die kleine Stechardin, München/Wien, Carl Hanser Verlag, 1994.

5. Benjamin, Deutsche Menschen, S.153.

6. Vgl. Tanja Reinlein, Der Brief als Medium der Empfindsamkeit, Würzburg, Königshausen \& Neumann, 2003.

7. Goethes Ehe in Briefen, hrsg. und eingel. von Hans Gerhard Gräf, Frankfurt a.M../Leipzig, Insel, 1994, hier S. 23. 
mit Christiane Vulpius ist dafür ein reizendes Beispiel. Christianes lautmalerisch am Mündlichen orientierte Orthographie streift häufig den Bereich des völlig Unverständlichen; Goethe schreibt seinem „Bettschatz“ im August 1792 während der Zeit der weltgeschichtlichen Wende von Valmy aus Longwy über die „gepflanzten Kohlrüben" ${ }^{8}$ und so geht es fort. Wir wollen das nicht loben, denn wir kennen den Preis, aber als Kontrastfolie, um die Tonart der Brautbriefe im oben genannten Sinne zu bestimmen, ist Goethes Korrespondenz mit seiner späteren, viel späteren Gattin durchaus geeignet.

Was den Stil der Briefe anlangt, datieren die Epistomologen die Durchsetzung eines neuen „natürlichen“ Briefstils in der Kommunikation zwischen Braut und Bräutigam zumeist mit Gellerts Briefe, nebst einer praktischen Abhandlung von dem guten Geschmacke in Briefen von $1751 .^{9}$ Geradezu Modellcharakter für den neuen, empfindsamen Briefstil erhielt der im gleichen Jahrzehnt entstandene Briefwechsel zwischen Klopstock und Meta Moller, in dem die reichlich aufs Rokokokleid fließenden Tränen nicht mehr als Zeichen der Schwäche, sondern der Gefühlstiefe gelesen werden wollen. ${ }^{10}$ Bekanntlich wird noch im 1774 erschienenen Werther der Moment der größten Nähe zwischen Werther und Lotte mit der Evozierung Klopstocks markiert. Den demonstrativ-gefühlsseligen Ton der Brautbriefe einfach durch die internationale Strömung der Empfindsamkeit erklären zu wollen, wäre tautologisch. Vor allem aber wäre auf diese Weise nicht erklärt, warum er weit über die entsprechende Epoche der sechziger und siebziger Jahre des 18. Jahrhunderts hinaus modellprägend wirkt, wie auf unnachahmlich komische Weise Kellers Erzählung Die missbrauchten Liebesbriefe aus der Novellensammlung Die Leute von Seldwyla vorführt. Dort hat der Viggi Störteler, ein in behaglichen, wenn auch nicht glänzenden Verhältnissen lebender Kaufmann und Freizeitdichter eine unselige Liebe zu „schweren Büchern“ und „verworrenen Unterhaltung[en].“ ${ }^{11}$ So beschließt er, seine Gattin „zu erhöhen und zu seiner Muse zu machen.“ ${ }^{12}$ Er entwirft einen „Bildungsplan“ und ermutigt sie zu Höherem: „[...] kehre Deine höhere Weiblichkeit hervor, lasse voll und rein die Harmonien ertönen, die in Dir schlafen müssen [...] Kurz, merke auf den Ton und Hauch in meinen Briefen und richte Dich danach, mehr sag' ich nicht." ${ }^{13}$ Die willige, aber für derlei Unsinn viel zu fest geerdete Gattin überstellt die in falscher Tonart gehauchten, aber Antwort heischenden Briefe ihres handlungsreisenden Gatten an einen benachbarten, heimlich in sie verliebten Lehrer und sendet dessen an sie gerichtete Antworten als ihre eigenen an den

8. Ibid., S. 60.

9. In Christian Fürchtegott Gellert, Gesammelte Schriften, kritische, kommentierte Ausgabe, hrsg. von Bernd Witte, Bd. 4, Berlin/ New York, de Gruyter, 1989, S. 105-221.

10. Meta Klopstock. Briefwechsel mit Klopstock, ihren Verwandten und Freunden, 3 Bde., hrsg. von Hermann Tiemann, Hamburg, Maximilian-Gesellschaft, 1956. Vgl. dazu Gérard Dautzenberg, „Mon coeur aurait tant de choses à vous dire.“ Meta et Klopstock, un couple célèbre de la littérature allemande, Paris, Sedes, 1990.

11. Gottfried Keller, Kleider machen Leute/ Die Leute von Seldwyla, 2. Teil, 3. Aufl., Herrsching, Pawlak, 1970, S. 80.

12. Ibid., S. 88.

13. Ibid., S. 92. 
literaturbeflissenen Gatten weiter, bis der Schwindel auffliegt, es zur Scheidung kommt und der Amateurdichter durch ein ebenso dummes wie sentimentalisches neues Eheweib gestraft wird.

Die Novelle erscheint 1873 als eine Art Komödiennachspiel zur sentimentalischen Gefühlstragik des 18. Jahrhunderts, die nur in dieser Form der schlechten Kopie noch möglich erscheint. Tatsächlich gibt es seit dem ausgehenden 19. Jahrhundert kaum noch Brautbriefe im erläuterten Sinne. In den letzten Jahrzehnten bekannt gewordene Ausnahmen sind der umfangreiche Briefwechsel von Sigmund Freud und Martha Bernays ${ }^{14}$ sowie der zwischen Dietrich Bonhoeffer und Maria von Wedemeyer ${ }^{15}$, der erste durchaus noch im 19. Jahrhundert verwurzelt, der zweite durch die Gefängnismauern der nationalsozialistischen Schergen in die Distanz gezwungen, die im 18. Jahrhundert die Verlobungspraxis erzwang, Ausnahmen alle beide, nur darin gewöhnlich, dass wir im allgemeinen nur von den Briefen von Verlobten erfahren, von denen ein Teil, der männliche fast immer, Berühmtheit erlangte.

Auch Gert Mattenklott, im Verein mit Heinz und Hannelore Schlaffer, datiert in der als Fortsetzung von Benjamins Band geplanten Sammlung Deutsche Briefe (1988) die Zeit der Brautbriefe von „1750 bis 1900 etwa“. ${ }^{16}$ Den drei Herausgebern erscheinen diese Briefe, die doch intim sein wollen, zumeist „verlegen und abstrakt“, was sich dadurch erkläre, dass sich die Verlobten häufig gar nicht kennten. An die Stelle des Austauschs trete da häufig die Schulmeisterei des Mannes. Überhaupt seien die entsprechenden Briefe „seltsam gleichförmig. Was doch das Individuelleste sein sollte, das Liebesverhältnis zweier Personen, entpuppt sich dem indiskreten Blick der Nachwelt als das Allgemeinste, als das Herrschaftsverhältnis der Geschlechter.“"17

Sicher, in den Brautbriefen wird vom Blatt geliebt, sicher, es geht häufig um die Erziehung der Braut, sicher zeigen sie auch die Asymetrie der Geschlechterverhältnisse, aber „verlegen und abstrakt“ sind diese Briefwechsel nicht immer - sondern häufıg in vielfältiger Hinsicht als Ausdruck von Kämpfen um Hegemonie lesbar, im Land, im Geist und in den Verhältnissen zwischen den Brautleuten. An zwei Beispielen, dem von Herder und Caroline Flachsland sowie von dem zwischen Kleist und Wilhelmine von Zenge ${ }^{18}$, ließe sich das hervorragend zeigen, freilich nicht gründlich im hier gesetzten Rahmen. Ich komme also hier nur zum ersten Beispiel und selbst im Falle der Brautbriefe zwischen Herder

14. Sigmund Freud und Martha Bernays, Die Brautbriefe, hrsg. von Gerhard Fichtner/ Ilse Grubrich-Simitis/Albrecht Hirschmüller, Frankfurt a.M., Fischer, 2013 ff. [5 Bde. geplant, 2 bisher erschienen].

15. Brautbriefe Zelle 92. Dietrich Bonhoeffer - Maria von Wedemeyer 1943-1945, hrsg. von RuthAlice von Bismarck/ Ulrich Kabitz; mit einem Nachwort von Eberhard Bethge, München, Beck, 1992.

16. Deutsche Briefe 1750-1950 (Anm. 2), S. 297.

17. Ibid., S. $297 \mathrm{f}$.

18. Vgl. die gründliche Darstellung durch Günter Blamberger, Heinrich von Kleist. Biographie, Frankfurt a.M., Fischer, 2011, S. 84-116. Sie bedürfte freilich an manchen Stellen einer eindringlichen Diskussion. 
und Flachsland muss ich mich auf einen Brief beschränken. Ich habe nicht den schon viel interpretierten ersten Brief Herders vom 25. August 1770 ausgewählt, sondern den vom 22. Juni 1771. Ungekürzt umfasst er vier Druckseiten. ${ }^{19}$

$\mathrm{Zu}$ den Voraussetzungen nur das Folgende: Der 1744 geborene Herder ist, vor allem auf Grund seiner Fragmente über die neuere deutsche Literatur und der Kritische[n] Wälder schon ein bekannter Autor, als er nach seiner Frankreich-Reise als Fürstenbegleiter im August 1770 in Darmstadt eintrifft. Am Hofe wurde Herder mit Johann Heinrich Merck bekannt, vor allem aber mit der zwanzigjährigen Caroline Flachsland (1750-1809), die im Hause ihrer Schwester lebte. Merck, Leuchsenring und ein Kreis empfindsamer junger Damen bürgerlicher wie adeliger Herkunft bildeten am Darmstädter Hof den literarisch interessierten Freundeskreis der „Darmstädter Empfindsamen“, die einen leidenschaftlichen Klopstock-Kult betrieben. Nach Herders Predigt am 19. August 1770 lernt er Caroline kennen, liest ihr in den nächsten Tagen aus Klopstock vor, der lange auch sein Lieblingsdichter war - und sechs Tage später schreibt er ihr den ersten Brief, in dem er ihr seine Liebe gesteht und der den Auftakt zu ihrer Brautkorrespondenz bildet. Zwei Tage später folgt seine Abreise, zunächst nach Karlsruhe, dann nach Straßburg mit den bekannten Folgen für die Entwicklung des Sturm- und Drang allgemein und Goethes Insonderheit. 1771 trat Herder dann eine Stelle in Bückeburg an, als Oberprediger, Gelehrter, als Hofmann, während Caroline in Darmstadt auf den Postboten warten musste.

Der Brief vom 22. 6. 1771 setzt brautbriefspezifisch mit Superlativen, aber durchaus launig ein:

Was machen Sie liebste, unschätzbare Freundin? Sind sie krank, sind Sie todt, sind sie böse, dass Sie so schweigen, oder denken Sie wieder, dass mein säumiger Briefwechsel zeige, wie gern ich die Hände aus dem Spiel haben wolle. ${ }^{20}$

Die Hände aus dem Spiel, das bezieht sich wohl auf Herders hartnäckige Weigerung, ein Eheversprechen abzugeben, an dem Caroline ebenso hartnäckig gelegen war. ${ }^{21}$ Caroline ist Waise, stammt aus einer mittellosen Beamtenfamilie mit drei schönen Töchtern, von denen die erste Mätresse des Fürsten von Hessen-Darmstadt war, in dieser Funktion ihre ganze Familie ernährte, bis sie schließlich geistesverwirrt endete und die Familie deshalb verarmte. Die zweite Tochter war unglücklich verheiratet mit dem höchsten Beamten des Darmstädter Hofs, Geheimrat von Hesse, in dessen Haushalt die dritte, Karoline eben, lebte, als Herder in Darmstadt war. Caroline, wusste also aus familiärer Erfahrung um

19. Johann Gottfried Herder, Briefe, Zweiter Band, Mai 1771 - April 1773, bearb. von Wilhelm Dobbek/ Günter Arnold, Weimar, Hermann Böhlaus Nachfolger, 1977, S. 34-39. Die methodischen Probleme der Isolierung eines Briefzeugnisses aus einem dichten, über drei Jahre währenden Briefwechsel können hier nicht diskutiert werden.

20. Ibid., S. 34 .

21. Ulrike Prokop, „Die Suche nach dem guten Leben. Zum Briefwechsel zwischen Caroline Flachsland und Johann Gottfried Herder“, in Márcia de Figuiredo/Sabine Klompaß (Hrsg.), Frauke Stübing, Kassel, kassel university press, 2009, S. 15-49, hier S. 34 [Kasseler Beiträge zur Erziehungswissenschaft, hrsg. vom Direktorium des Instituts für Erziehungswissenschaft, Bd. 2]. 
die Gefahren der adeligen Mätressenrolle wie der neigungsfreien Vernunftheirat. Sie sah die Chance, die ein Beziehungstypus, wie ihn Klopstock mit Meta lebte, für die Frau bot und begriff schnell, dass diese für sie die Gestalt Herders hatte. Wenn der nur heiratswillig gewesen wäre...

Herder umschifft in unserem Brief anfangs das Problem und verspricht, von den „Sachen zusammen [zu] plaudern, von denen ich so lange schon mit meiner Einzigen und Ersten Freundin reden wollte." Die Plauderei kommt zunächst höchst banal auf „grässlichste Zahnschmerzen“ und anderes „Nichtwohlbefınden“ zu sprechen, kommt dann auf „Fräulein Sternheim“, auf die wir noch zurückkommen müssen, um darauf ein „Bekenntnis des Herzens“22 anzukündigen, das sich aber, wie man dann sieht, keineswegs auf Heiratspläne bezieht, sondern auf seinen Eindruck vom Bückeburger Grafen, der ihn doch ehrenvoll berufen hatte. Das als Bekenntnis Angekündigte kommt als politische Einschätzung von eher seltener Eindeutigkeit und Radikalität nebenbei daher: „Uebrigens herrscht hier in dem kleinen Ländchen ein solcher Despotismus, eine solche kriechende u. garstige Kleinheit, als ich selbst an den Despotischten Orten nicht gefunden. ${ }^{23}$ So deutlich wurde es selten geschrieben. Das Thema wird aber scheinbar leichthin wieder verlassen, um mit routinierter Bescheidenheitsgeste darauf zu verweisen, dass der Schreiber nicht nur Zahnschmerzen bekommen hatte, sondern immerhin auch den Preis der Berliner Akademie für seine Schrift über den Ursprung der Sprache. Freilich „nur Schade, Schade, dass es mir durchaus an Gesellschaft zur Bildung u. zur Empfindung fehlt. Da, meine liebste Freundin, muß mir Ihr Briefwechsel Alles ersetzen und ihr Bild ist wenigstens ein schöner Trugeschatten, der mich, auch jetzt in den Intervallen meiner Höllenschmerzen nicht allein lässt." ${ }^{24}$

Wir sollten das scheinbar nur dahin Geplauderte beim Worte nehmen. Die deutschen Kleinstaaten sind despotisch und was die bürgerliche Intelligenz dagegen aufzubieten hat, ist Bildung und Empfindung. Bildung und Empfindung, aber nicht in vergesellschafteter Form. Es fehlt weithin noch an dem, was Habermas später die bürgerliche Öffentlichkeit nennen wird, in Bückeburg mit seinen kaum 3000 Einwohnern besonders. An die Stelle der Öffentlichkeit tritt die bescheidene des Briefwechsels, der Briefwechsel mit einer Frau, die hier seltsamer Weise „ein schöner Trugeschatten“ genannt wird. Im Worte liegt das Eingeständnis, dass die Adressatin des Brautbriefs im ursprünglichen Wortsinne imaginär ist, imaginär und schön und Trug aber auch, auf dass sie für im Stande gehalten werden könne, die fehlende Gesellschaft als Adressatin der Bildung und Empfindung zu ersetzen.

Nirgendwo sind die Motive für die Idealisierung der Braut in den Brautbriefen besser zu fassen als an dieser Stelle. Und man sieht auch: Sie brauchen die Liebe dieser Bräute, unsere gebildeten Feinde des Despotismus, denn diese Liebe ist ihnen Vorschein und Versprechen einer höheren, menschheitlichen.

22. Herder, Briefe, S. 34 und 35.

23. Ibid., S. 36.

24. Ibid., S. 37. 
Die leider in der Wirklichkeit fortbestehende Differenz sozialer Position wird mit empfindsamem Pathos verkittet:

Je mehr ich die Menschheit lieben lerne, o Mädchen, desto mehr lasset es uns seyn! [nämlich menschlich, K.H.G.] in jeder Thräne, u. Schwachheit derselben sitzt mehr als ein Engel! Lieben Sie mich, u. ihr Bild voll Sittsamkeit und Liebreiz u. Zucht u. Adel u. Aufopferung u. Ergebenheit u. Zärtlichkeit - süßes Bild sei das Brustbild meines Herzens; auf Dich will ich $\mathrm{Kuß}$ und Hand legen $u$. einst sterben! ${ }^{25}$

Lesen wir den routinierten Gefühlsausdruck genau: geküsst wird ein Bild, kein Mensch, um die Hand wird nicht angehalten, sondern besitzergreifend wird sie belegt und gestorben wird dermaleinst auch auf einem Bild der Frau, deren ideale Eigenschaften in parataktischer Unendlichkeit aufgereiht werden können. Wir wissen schon: das Bild ist ein Trugbild.

Direkt darauf markiert ein Gedankenstrich eine scheinbar neue Wendung des Plauderbriefs: Auf Carolines Erzählung in ihrem vorausgehenden Brief, der bejahrte Dichter Gleim habe sie bei seinem Besuch der Darmstädter Empfindsamen in den Arm genommen, erinnert sich Herder daran, wie er sie einst auch so gehalten: „wie sie da in Gleims Arm lagen! Ach, an meinem lagen sie auch! Standen, wie die Liebegöttin auf dem Gerüst vor dem Pygmalion; so sie am Fenster, mit dem liebescheuen und liebetrunkenen Blick der Seele! Wollen Sie ewig mein Mädchen seyn?" ${ }^{26}$ Herder erinnert sich hier nicht ohne Eifersucht an eine Art Urszene seiner Liebe zu Caroline und kommt dabei mit dem „sitzen“ und „stehen“ etwas durcheinander. Stellen wir uns die Szene vor: Caroline steht am Fenster, von dessen Rahmen sorgfältig gefasst wie ein Kunstwerk. Sie steht gegen das Licht - und ist also abermals Schatten. Aller konkreten Züge entwirklicht und zum Kunstwerk gerahmt, kann nun Göttlichkeit auf sie projiziert werden. Der Entwirklichung folgt die mythologische Erhöhung auf dem Fuße.

Man muss kein Psychoanalytiker sein, um zu entschlüsseln, warum sich Herder hier als Pygmalion sieht. In Ovids Text schafft der von den Frauen enttäuschte Pygmalion eine weibliche Marmorstatue nach seinen Vorstellungen von Reinheit und Schönheit, verliebt sich in sie und wünscht sich von Venus, seine künftige Frau solle so beschaffen sein wie die Statue, ein Wunsch, der schließlich in Erfüllung geht. Im 18. Jahrhundert bekommt die Statue den Namen Galatea. Es ist ein Jahrhundert, in dem es in Deutschland wie in Frankreich zahlreiche Varianten der Geschichte gibt, die als die mythologische Erzählung vom männlichen Modellierungswunsch gelten darf, als Erzählung von der Phantasie, sich eine Frau gleichsam zu backen.

Nun ist Herder kein Bildhauer, sondern Literaturkritiker. Seine Modellierung kann nicht mit Hammer und Meißel erfolgen, sondern durch Bildung, ein Wort, das ja durchaus semantische Verwandtschaft mit dem der bildhauerischen Modellierung aufweist. Durch Bildung vermittels der Lektüre - und so handeln denn auch drei große Passagen unseres Briefes von einem eben erschienenen Text, 
den beide Brautleute gelesen haben und der Caroline als Modell anempfohlen wird, die Geschichte des Fräulein von Sternheim, geschrieben von Sophie von La Roche, herausgegeben von Wieland. Hier wird die geteilte Lektüreerfahrung geradezu zum Ausweis einer Gefühlsgemeinschaft:

So haben sie die Sternheim so gefühlt, u. eben wie ich gefühlt! 0 gemeinschaftlicher Schutzgeist unsres Lebens, wenn wir sie einst zusammen lesen werden. ${ }^{27}$

Der Bezug auf den Roman über das Fräulein von Sternheim, so hat Ulrike Prokop unter Auswertung aller entsprechenden Stellen in unserem Briefwechsel überzeugend nachgewiesen, markiert freilich nicht nur einen Fonds von Gemeinsamkeiten in Inhalt und Rezeption, sondern dient Herder auch dazu, bei seiner Braut die rechte Art von weiblicher Lektüre durchzusetzen, die nicht kritisch sein darf, weil das einem Weibe nicht ziemt, sondern identifikatorisch, er will Caroline „eben so handeln [..] sehen“ wie das Fräulein. ${ }^{28}$ An Merck hingegen richtet er am 6.11.1770 einen Brief über den Roman von La Roche, in dem es um Fiktionalität und den Kunstcharakter des Werkes geht. Kunstkritische Diskussion für die Männer, Gefühlsgemeinschaft mit und literarische Identifikationsmodelle für die Frauen. ${ }^{29}$

Ich muss den Blick auf die literaturgestützten Modellierungstrategien hier abbrechen und kann auch nicht, wie im Zusammenhang mit Kleist beabsichtigt, auf das einflussreichste dieser Modelle zu sprechen kommen, Rousseaus $E_{\text {Émile }}{ }^{30}$ damit uns noch ein ganz klein wenig Platz bleibt für den Aspekt der Übersteigerung. In unserem Brief finden sich ja höchst banale, stilistisch normal gestimmte Passagen neben stilistisch und inhaltlich hoch gesteigerten. Die Verteilung, der hier nicht systematisch nachgegangen werden kann, ist nicht zufällig. Kehren wir noch einmal zur Pygmalion-Passage zurück, die gefolgt wird von Zitaten aus Carolines Briefen, die Herder offenbar missfallen haben, weil sie sein „Trugbild“ störten. Dazu gehörte Carolines Einwurf „wir sind keine Kinder mehr!“" ${ }^{31}$, der sich auf das Übermaß von Herders epistolären Seufzern und Herzensergießungen bezieht, vor dem Hintergrund des gesamten Briefwechsels aber zweifellos auch darauf, dass Herder sie als Mädchen will, aber zögert, sie zur Frau zu nehmen. Carolines schriftlicher Widerstand löst eine wahre Flut höchst erregter Ausrufe aus:

Ich bin unglücklich! Die süße Wolke, die sich so edel u. wahrhaft über meinen Horizont zieht, um freilich den Sonnenstral der Freude zu ersticken, aber desto länger u. ewiger den süßen Schatten zu machen, der holde Wehmuth und Sehnsucht heisst - die Wolke wird mir

27. Ibid.

28. Ibid., S. 35

29. Vgl. dazu die eingehende Analyse bei Ulrike Prokop, „Die Suche nach dem guten Leben“, S. $38-42$.

30. Vgl. dazu Blamberger, Heinrich von Kleist, S. 105-108.

31. Herder, Briefe, S. 38. 
Zeichen Kains! u. Abscheu! - Ist denn kein Seufzer (Gott! kennten sie meinen Zustand) ohne Kindheit und Falschheit möglich! ${ }^{32}$

Der Einwand gegen Übersteigerung löst stilistisch übersteigerte Übersteigerung aus. Inhaltlich bleibt da nur noch ein Weg höher hinaus, den Herder nicht zögert, zu gehen: „0 seyn Sie, meine Vorbitterin bei Gott, süße Liebe!“”33

Der Widerstand der despotischen Landesfürsten, die Abwesenheit einer gebildeten Gesellschaft verweisen die ehrgeizige Intelligenz um 1770 auf die Frau, auf ein idealisiertes Trugbild von Frau, das modelliert werden muss nach Maßgabe literarischer Vorgaben. Wo sie sich mit eigenen Interessen zu Worte meldet, wird ihre Stimme von den Seufzern einer Gefühlskultur übertönt, die, so wie die Dinge in Deutschland liegen, zugleich eine Bedingung ihrer partiellen Emanzipation war. 